\title{
The proposed "Waldmeier discontinuity": How does it affect to sunspot cycle characteristics?
}

\author{
Alejandro Jesús Pérez Aparicio, José Manuel Vaquero*, and María Cruz Gallego \\ Departamento de Física, Universidad de Extremadura, 06800 Mérida, Spain \\ * corresponding author: e-mail: jvaquero@unex.es
}

Received 24 February 2012 / Accepted 1 August 2012

\begin{abstract}
Recently, Svalgaard has proposed that Waldmeier introduced a discontinuity in the International Sunspot Number (ISN) around 1945. In this paper, we study some characteristics of the sunspot cycle using the classical ISN and the proposed version derived from the "Waldmeier discontinuity". We conclude that this proposed version does not significantly improve the statistics of the characteristics of solar cycle.
\end{abstract}

Key words. solar activity - solar cycle - sunspot - space climate - historical records

\section{Introduction}

The most important index of solar activity is the Wolf or Zürich Sunspot Number (now called the International Sunspot Number, $I S N$ ) that was defined by Rudolf Wolf in the 19th century. Currently, the ISN is provided by the Solar Influences Data Analysis Center (SIDC) in Brussels, Belgium (Clette et al. 2007). However, Hoyt \& Schatten (1998) made a comprehensive work of recovery of sunspot data and defined a new index based on these observations: Group Sunspot Number (GSN). This index is designed to have similar values that ISN had in the period 1873-1986. GSN only depends on the number of sunspot groups while $I S N$ depends on the number of groups and the number of individual spots.

Some authors have compared both sunspot number series from different points of view: spectral characteristics (Faria et al. 2004; Li et al. 2005; Getko 2006; Li \& Liang 2010) and sunspot cycle characteristics (Hathaway et al. 2002). Recently, Svalgaard (2010) has studied the ratio of ISN and $G S N$ showing an apparent discontinuity in 1945 just when Max Waldmeier took over the production of the ISN. According to Svalgaard (2010), Waldmeier introduced an upward jump in the sunspot number in 1945. Therefore, Svalgaard (2010) proposed that all values before 1945 were increased by $20 \%$. Note that when the SIDC took over the production of ISN (just after Waldmeier), it strove to maintain continuity with the values of Waldmeier. Therefore, it is possible to construct a Modified International Sunspot Number (MISN) increasing by $20 \%$ for all values of the $I S N$ prior to 1945 .

In this paper we study the behavior of different sunspot numbers (GSN, ISN and MISN) from the point of view of the solar cycle characteristics. First, we consider solar cycles (SC) 1-22. Then, we made the same study only for $S C 10-22$, which is the best quality period of this series (Vaquero 2007) because the annual number of days without records is zero.

\section{Data}

In the present study, we employ the usual smoothed monthly mean values (13-month running mean) of GSN, ISN and MISN, computed from the monthly mean sunspot numbers available from Solar Influences Data Analysis Center (SIDC, http://sidc.oma.be/) and National Geophysical Data Center (NGDC, http://www.ngdc.noaa.gov/stp/solar/ssndata.html). From these values, we computed the following parameters for each solar cycle: ascending time $\left(T_{\mathrm{a}}\right)$, descending time $\left(T_{\mathrm{d}}\right)$, amplitude $(A)$, period of the preceding cycle $\left(P_{\text {pre }}\right)$ and preceding cycle minimum $\left(M_{\text {pre }}\right)$. They are listed in Tables 1-3 (GSN, ISN and MISN, respectively). We use data for $S C 1-22$, i.e. the common period of this three sunspot numbers.

\section{Results}

In order to compare the three sunspot numbers (GSN, ISN and $M I S N$ ), we are going to consider four well-known characteristics of solar cycle: "Waldmeier Effect" (the anticorrelation between the cycle amplitude and the length of the ascending phase of the cycle, Waldmeier 1939), "Amplitude-Period Effect" (the anticorrelation between the cycle amplitude and the length of the previous cycle from minimum to minimum, Chernosky 1954; Wilson et al. 1998), "Amplitude-Minimum Effect" (the correlation between cycle amplitude and the activity level at the previous minimum, Wilson et al. 1998) and "Amplitude-Descending Time Effect" (the correlation between cycle amplitude and the descending time of previous solar cycles, Du \& Du 2006).

Note that also other correlations have been described in the literature. We can cite, for example, that (a) the amplitude of $S C n$ is inversely related to the period of the $S C n$ (Charbonneau \& Dikpati 2000; Hathaway et al. 2002) and (b) the amplitude of $S C n$ is related to the period of the $S C n-3$ (Solanki et al. 2002; Du et al. 2006). 
Table 1. Solar cycle parameters used in this study for GSN.

\begin{tabular}{|c|c|c|c|c|c|c|}
\hline \multirow[b]{2}{*}{$S C$} & \multicolumn{6}{|c|}{$G S N$} \\
\hline & $T_{\mathrm{a}}$ (months) & Rise rate $\left(\right.$ months $^{-1}$ ) & $T_{\mathrm{d}}$ (months) & $A$ & $P_{\text {pre }}$ (months) & $M_{\text {pre }}$ \\
\hline 1 & 71 & 1.0 & 59 & 71.3 & & 4.6 \\
\hline 2 & 41 & 2.6 & 69 & 106.5 & 130 & 2.5 \\
\hline 3 & 48 & 1.7 & 57 & 79.5 & 110 & 5.8 \\
\hline 4 & 43 & 2.1 & 145 & 90.5 & 105 & 0 \\
\hline 5 & 20 & 2.6 & 107 & 51.1 & 188 & 3.5 \\
\hline 6 & 75 & 0.4 & 78 & 31.5 & 127 & 0 \\
\hline 7 & 81 & 0.8 & 47 & 64.4 & 153 & 0.1 \\
\hline 8 & 40 & 2.9 & 76 & 116.8 & 128 & 5.2 \\
\hline 9 & 64 & 1.5 & 85 & 93.2 & 116 & 8.3 \\
\hline 10 & 58 & 1.5 & 79 & 85.8 & 149 & 1.7 \\
\hline 11 & 42 & 2.4 & 97 & 99.9 & 137 & 4.6 \\
\hline 12 & 63 & 1.1 & 71 & 68.2 & 139 & 1.8 \\
\hline 13 & 47 & 2.0 & 96 & 96 & 134 & 5.6 \\
\hline 14 & 49 & 1.3 & 89 & 64.6 & 143 & 2.2 \\
\hline 15 & 49 & 2.3 & 73 & 111.3 & 138 & 1.7 \\
\hline 16 & 58 & 1.4 & 62 & 81.6 & 122 & 6.6 \\
\hline 17 & 43 & 2.9 & 83 & 125.1 & 120 & 4.4 \\
\hline 18 & 40 & 3.6 & 81 & 145.2 & 126 & 7.9 \\
\hline 19 & 47 & 4.0 & 75 & 186.1 & 121 & 3.1 \\
\hline 20 & 72 & 1.5 & 69 & 109.3 & 122 & 10 \\
\hline 21 & 40 & 3.9 & 80 & 154.2 & 141 & 12.8 \\
\hline 22 & 44 & 3.5 & & 153 & 120 & 11.1 \\
\hline
\end{tabular}

Table 2. Solar cycle parameters used in this study for ISN.

\begin{tabular}{|c|c|c|c|c|c|c|}
\hline \multirow[b]{2}{*}{$S C$} & \multicolumn{6}{|c|}{$I S N$} \\
\hline & $T_{\mathrm{a}}$ (months) & Rise rate $\left(\right.$ months $^{-1}$ ) & $T_{\mathrm{d}}$ (months) & $A$ & $P_{\text {pre }}$ (months) & $M_{\text {pre }}$ \\
\hline 1 & 75 & 1.2 & 59 & 86.5 & & 8.4 \\
\hline 2 & 40 & 2.9 & 69 & 115.8 & 134 & 11.2 \\
\hline 3 & 35 & 4.5 & 76 & 158.5 & 109 & 7.2 \\
\hline 4 & 41 & 3.4 & 122 & 141.2 & 111 & 9.5 \\
\hline 5 & 82 & 0.6 & 65 & 49.2 & 163 & 3.2 \\
\hline 6 & 70 & 0.7 & 83 & 48.7 & 147 & 0 \\
\hline 7 & 79 & 0.9 & 48 & 71.5 & 153 & 0.1 \\
\hline 8 & 40 & 3.7 & 76 & 146.9 & 127 & 7.3 \\
\hline 9 & 55 & 2.4 & 94 & 132 & 116 & 10.6 \\
\hline 10 & 50 & 2.0 & 85 & 98 & 149 & 3.2 \\
\hline 11 & 41 & 3.4 & 100 & 140.3 & 135 & 5.2 \\
\hline 12 & 60 & 1.2 & 74 & 74.6 & 141 & 2.2 \\
\hline 13 & 47 & 1.9 & 96 & 87.9 & 134 & 5 \\
\hline 14 & 49 & 1.3 & 89 & 64.2 & 143 & 2.7 \\
\hline 15 & 49 & 2.2 & 72 & 105.4 & 138 & 1.5 \\
\hline 16 & 56 & 1.4 & 65 & 78.1 & 121 & 5.6 \\
\hline 17 & 43 & 2.8 & 82 & 119.2 & 121 & 3.5 \\
\hline 18 & 39 & 3.9 & 83 & 151.8 & 125 & 7.7 \\
\hline 19 & 47 & 4.3 & 79 & 201.3 & 122 & 3.4 \\
\hline 20 & 49 & 2.3 & 91 & 110.6 & 126 & 9.6 \\
\hline 21 & 42 & 3.9 & 81 & 164.5 & 140 & 12.2 \\
\hline 22 & 34 & 4.7 & 82 & 158.5 & 123 & 12.3 \\
\hline
\end{tabular}

\subsection{Waldmeier effect}

We consider the Waldmeier Effect comparing the amplitude and the ascending time for each solar cycle. Figure 1 (upper panels) shows the relationship between both parameters for $S C$ 1-22 (left panel) and $S C$ 10-22 (right panel).

Using data from $S C$ 1-22, we obtain a high linear Pearson coefficient for ISN and MISN ( -0.741 and -0.758 , respectively). However, we obtain a low correlation coefficient for
$G S N$ (-0.381). These results vary significantly if we use data from the $S C$ 10-22, correlation coefficients were slightly lower for ISN and MISN (-0.669 and -0.666 , respectively) and a higher correlation coefficient for $G S N$ $(-0.509)$.

We note that the "Waldmeier Effect" is very weak in the $G S N$, as was already shown by Hathaway et al. (2002), and there are no significantly different values when we use ISN or MISN. 
A.J.P. Aparicio et al.: Waldmeier discontinuity: How does it affect to solar cycle characteristics?

Table 3. Solar cycle parameters used in this study for MISN.

\begin{tabular}{|c|c|c|c|c|c|c|}
\hline \multirow[b]{2}{*}{$S C$} & \multicolumn{6}{|c|}{$M I S N$} \\
\hline & $T_{\mathrm{a}}$ (months) & Rise rate $\left(\right.$ months $^{-1}$ ) & $T_{\mathrm{d}}$ (months) & $A$ & $P_{\text {pre }}$ (months) & $M_{\text {pre }}$ \\
\hline 1 & 75 & 1.4 & 60 & 103.8 & & 10.1 \\
\hline 2 & 39 & 3.6 & 69 & 138.9 & 135 & 13.4 \\
\hline 3 & 35 & 5.4 & 76 & 190.3 & 108 & 8.6 \\
\hline 4 & 41 & 4.1 & 122 & 169.4 & 111 & 11.5 \\
\hline 5 & 82 & 0.7 & 65 & 59 & 163 & 3.8 \\
\hline 6 & 70 & 0.8 & 83 & 58.4 & 147 & 0 \\
\hline 7 & 79 & 1.1 & 48 & 85.8 & 153 & 0.1 \\
\hline 8 & 40 & 4.4 & 76 & 176.3 & 127 & 8.8 \\
\hline 9 & 55 & 2.9 & 94 & 158.3 & 116 & 12.7 \\
\hline 10 & 50 & 2.4 & 85 & 117.6 & 149 & 3.8 \\
\hline 11 & 41 & 4.1 & 100 & 168.4 & 135 & 6.2 \\
\hline 12 & 60 & 1.5 & 74 & 89.6 & 141 & 2.7 \\
\hline 13 & 47 & 2.2 & 96 & 105.5 & 134 & 6 \\
\hline 14 & 49 & 1.6 & 89 & 77.1 & 143 & 3.2 \\
\hline 15 & 49 & 2.6 & 72 & 126.5 & 138 & 1.8 \\
\hline 16 & 56 & 1.7 & 65 & 93.8 & 121 & 6.7 \\
\hline 17 & 43 & 3.3 & 82 & 143 & 121 & 4.2 \\
\hline 18 & 39 & 3.9 & 83 & 151.8 & 125 & 9.3 \\
\hline 19 & 47 & 4.3 & 79 & 201.3 & 122 & 3.4 \\
\hline 20 & 49 & 2.3 & 91 & 110.6 & 126 & 9.6 \\
\hline 21 & 42 & 3.9 & 81 & 164.5 & 140 & 12.2 \\
\hline 22 & 34 & 4.7 & 82 & 158.5 & 123 & 12.3 \\
\hline
\end{tabular}
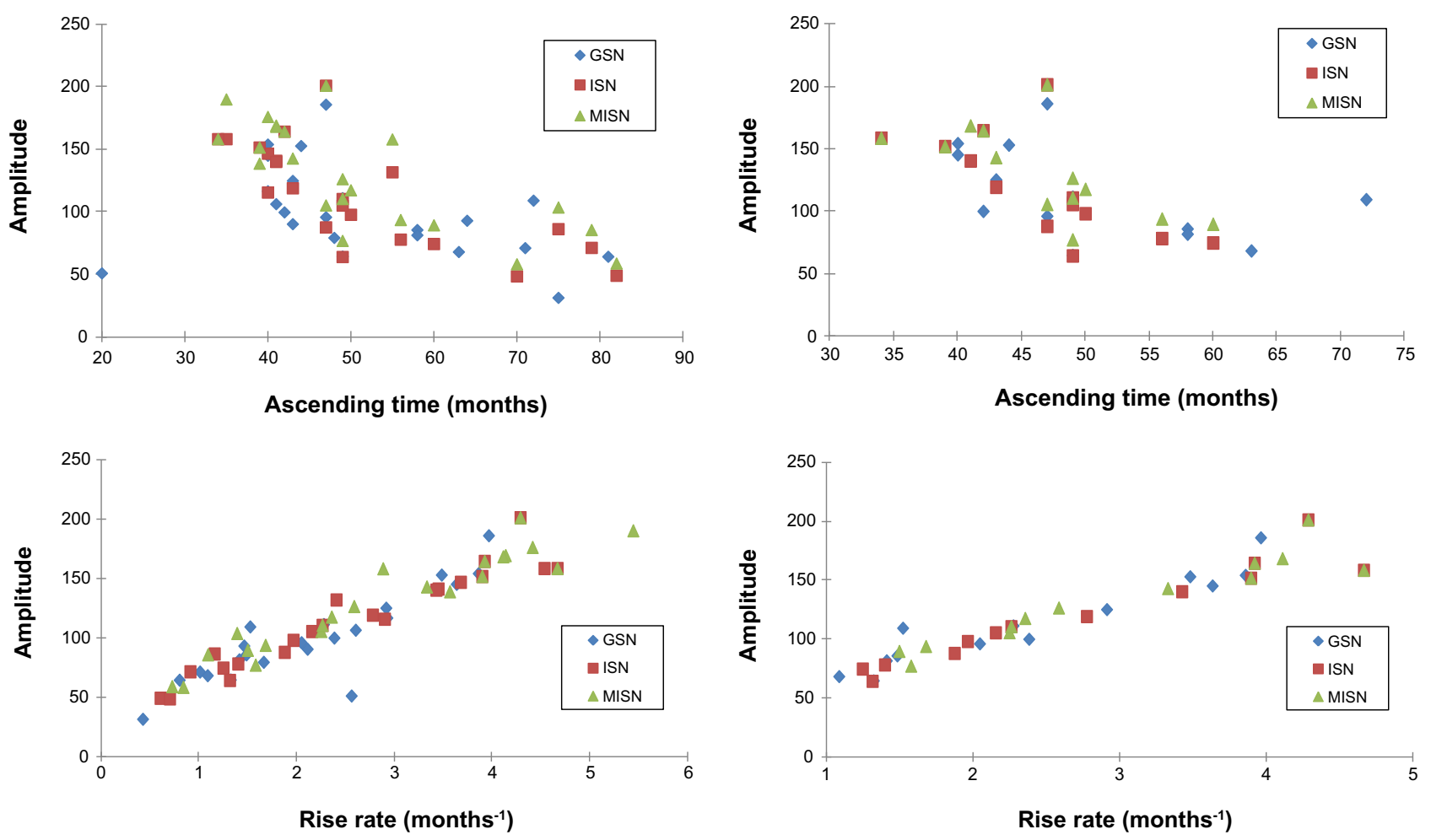

Fig. 1. Upper panels: scatter plot of the classical Waldmeier Effect for cycles 1-22 (left panel) and 10-22 (right panel). Lower panels: scatter plot of a variation of the Waldmeier Effect for cycles 1-22 (left panel) and 10-22 (right panel).

A variation of the "Waldmeier Effect" is the correlation between the cycle amplitude and the rise rate (Cameron \& Schüssler 2007; Dikpati et al. 2008; Karak \& Choudhuri 2011). We can also consider this variation of the "Waldmeier Effect". Figure 1 (lower panels) shows the relationship between both parameters for SC 1-22 (left panel) and SC 10-22 (right panel).
We obtain very high correlation coefficients for SC 1-22 using ISN, MISN and GSN $(0.956,0.951$ and 0.878 , respectively). If we use data of $S C 10-22$, we obtain similar correlation coefficients for ISN and MISN (0.954 and 0.942, respectively) and higher for $G S N(0.949)$. We note that the correlation in MISN is slightly weaker than in $I S N$. 

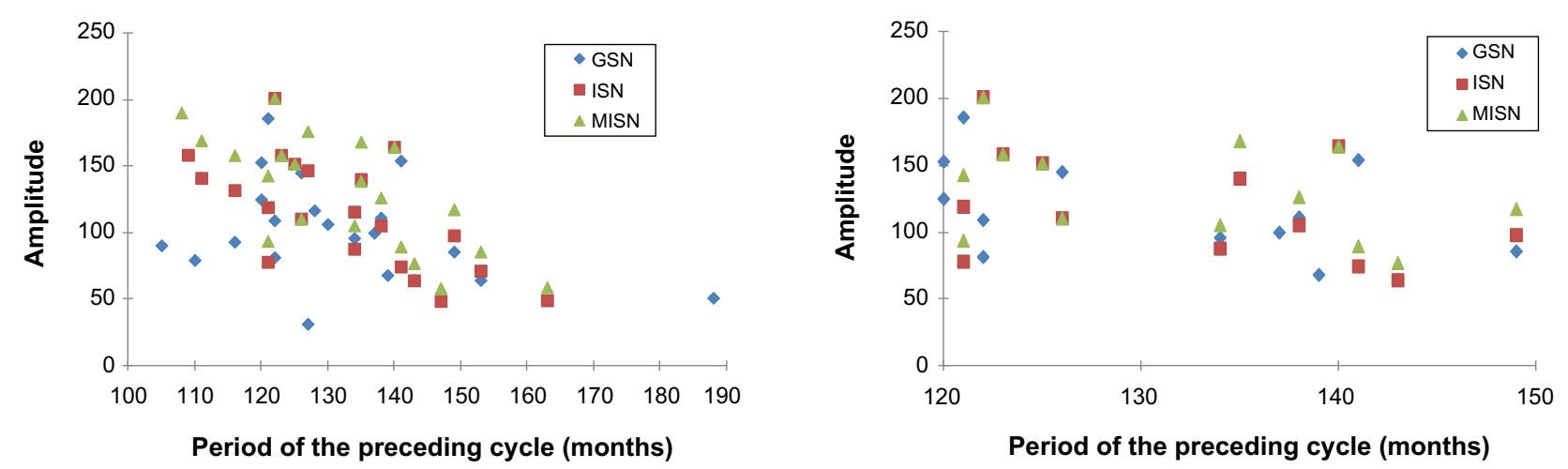

Fig. 2. Scatter plot of the Amplitude-Period Effect for cycles 1-22 (left panel) and 10-22 (right panel).
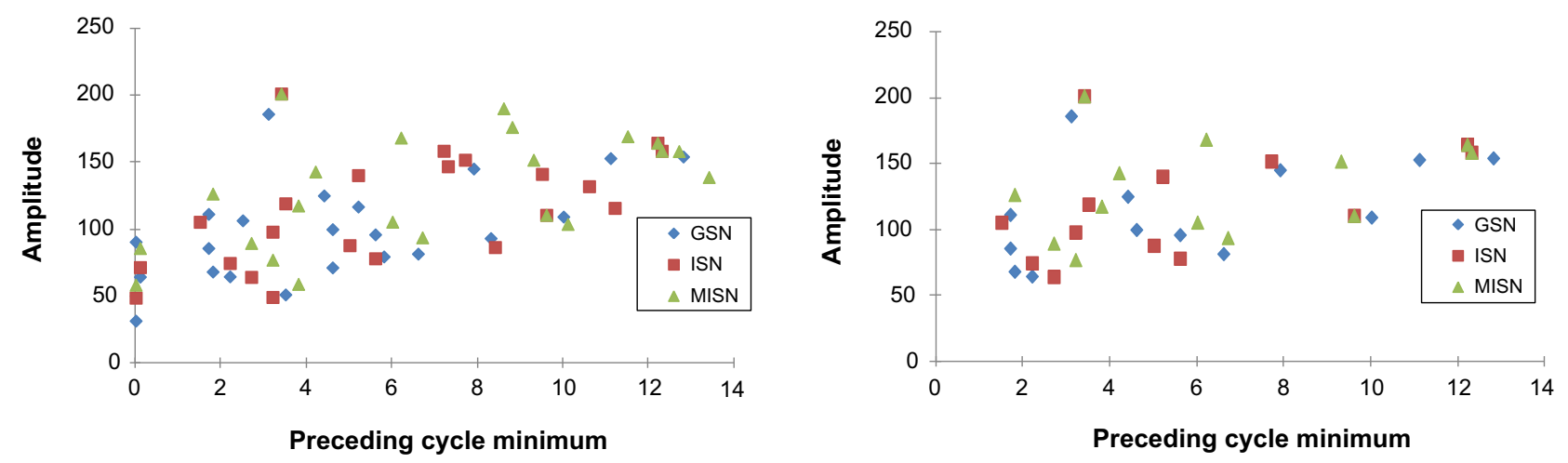

Fig. 3. Scatter plot of the Amplitude-Minimum Effect for cycles 1-22 (left panel) and 10-22 (right panel).
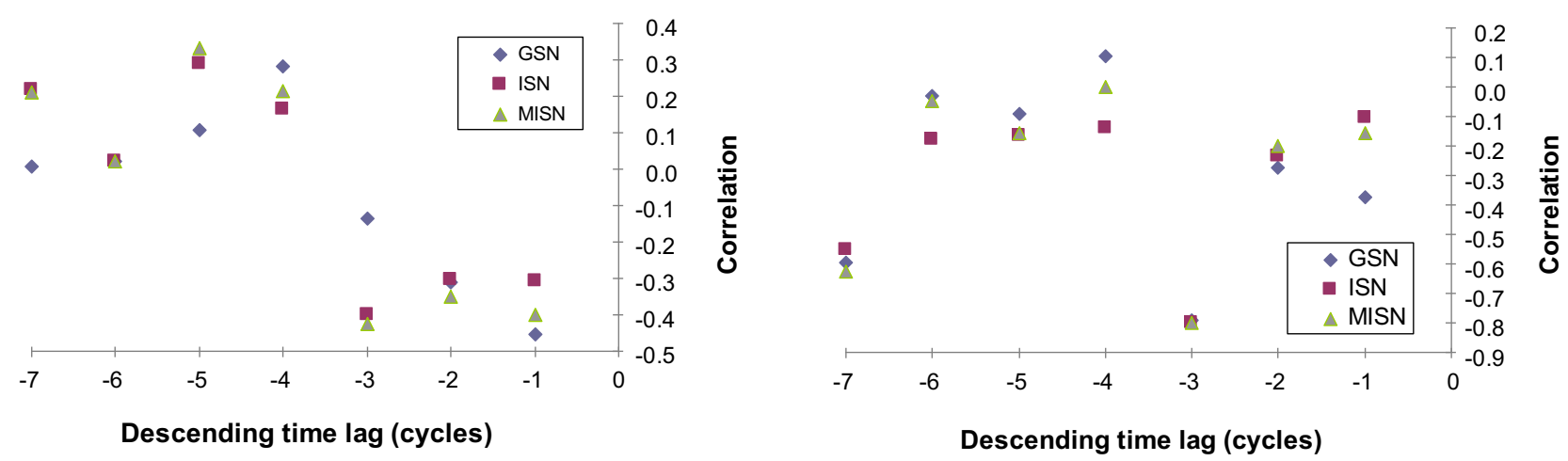

Fig. 4. Cross-correlation coefficient between amplitude and descending time at lags from one to seven cycles for cycles 1-22 (left panel) and $10-22$ (right panel).

\subsection{Amplitude-period effect}

The second studied characteristic is the Amplitude-Period Effect, that is considered by comparing the amplitude of a solar cycle and the period of the preceding solar cycle. Figure 2 shows the relationship between both parameters for $S C 1-22$ (left panel) and SC 10-22 (right panel).

Using data from $S C$ 1-22, we obtain a high correlation coefficient for $I S N$ and MISN ( -0.669 and -0.719 , respectively). Moreover, we obtain a low correlation coefficient for GSN (-0.358). However, correlation coefficients were lower for $I S N$ and MISN ( -0.419 and -0.376 , respectively) and higher for $G S N(-0.512)$ with data from the $S C 10-22$.
Therefore, "Amplitude-Period Effect" is very weak in the $G S N$, as was already shown by Hathaway et al. (2002) and Vaquero \& Trigo (2008). Results for $I S N$ and MISN are very similar. Note that Vaquero \& Trigo (2008) concluded that this relationship is only strongly significant in a statistical sense during the first half of the historical record of $I S N$. Moreover, it is considerably less significant for the GSN.

\subsection{Amplitude-minimum effect}

The Amplitude-Minimum Effect has also been considered. Figure 3 represents the solar amplitude versus the activity 
A.J.P. Aparicio et al.: Waldmeier discontinuity: How does it affect to solar cycle characteristics?

Table 4. Summary of correlation coefficients obtained in this study. Single asterisk indicates a $95 \%$ significant level. Double asterisks indicate a $99 \%$ significant level.

\begin{tabular}{|c|c|c|c|c|}
\hline & $S C$ & $I S N$ & MISN & $G S N$ \\
\hline Waldmeier Effect & $\begin{array}{r}1-22 \\
10-22\end{array}$ & $\begin{array}{l}-0.741^{* *} \\
-0.669^{* *}\end{array}$ & $\begin{array}{l}-0.758^{* *} \\
-0.666^{* *}\end{array}$ & $\begin{array}{l}-0.381^{*} \\
-0.509^{*}\end{array}$ \\
\hline Modified Waldmeier Effect & $\begin{array}{r}1-22 \\
10-22\end{array}$ & $\begin{array}{l}0.956^{* *} \\
0.954^{* *}\end{array}$ & $\begin{array}{l}0.951^{* *} \\
0.942^{* *}\end{array}$ & $\begin{array}{l}0.878 * * \\
0.949 * *\end{array}$ \\
\hline Amplitude-Period Effect & $\begin{array}{r}1-22 \\
10-22\end{array}$ & $\begin{array}{l}-0.669^{* *} \\
-0.419\end{array}$ & $\begin{array}{l}-0.719^{* *} \\
-0.376\end{array}$ & $\begin{array}{l}-0.358 \\
-0.512 *\end{array}$ \\
\hline Amplitude-Minimum Effect & $\begin{array}{r}1-22 \\
10-22\end{array}$ & $\begin{array}{l}0.559^{*} \\
0.468^{*}\end{array}$ & $\begin{array}{l}0.536^{*} \\
0.311\end{array}$ & $\begin{array}{l}0.542^{*} \\
0.479^{*}\end{array}$ \\
\hline Amplitude-Descending Time Effect & $\begin{array}{r}1-22 \\
10-22\end{array}$ & $\begin{array}{l}-0.399 \\
-0.799 * *\end{array}$ & $\begin{array}{l}-0.424 \\
-0.801 * *\end{array}$ & $\begin{array}{l}-0.136 \\
-0.791 * *\end{array}$ \\
\hline
\end{tabular}

level at the previous minimum for $S C$ 1-22 (left panel) and $S C$ 10-22 (right panel).

We obtain low correlation coefficients between amplitude and the activity level at the previous minimum for $S C$ 1-22 using ISN, MISN and GSN (0.559, 0.536 and 0.542, respectively). If we use data of $S C 10-22$, we obtain lower correlation coefficients for ISN, MISN and GSN (0.468, 0.311 and 0.479, respectively). Note that the lowest value of the correlation coefficient is obtained for MISN.

\subsection{Amplitude-descending time effect}

In this section, we analyze the relationship between the cycle amplitude and the descending time of previous $S C$. Note that the amplitude of a solar cycle was found to be well correlated (correlation coefficient equal to -0.811) with the descending time three cycles earlier, in smoothed monthly mean sunspot numbers for $S C$ 8-23 (Du \& Du 2006). Therefore, this relationship could be useful for long-term solar activity predictions.

However, we do not find any significant correlation using data of SC 1-22 and lags from -1 to -7 (Fig. 4, left panel) for all considered sunspot numbers. In agreement with Du \& $\mathrm{Du}$ (2006), we found a negative correlation (-0.799) for lag equal to -3 using $I S N$ and data of SC 10-22 (Fig. 4, right panel). Moreover, we found almost the same result for MISN and $G S N(-0.801$ and -0.791 , respectively) for $S C 10-22$.

\section{Conclusions}

We have computed the correlation coefficients between some parameters that are related to several characteristics of the solar cycle. These results are summarized in Table 4. According to our analysis, the following conclusions have been reached:

1. The "Waldmeier Effect" and the "Amplitude-Period Effect" are much more apparent in the ISN and MISN than in GSN. The "Amplitude-Minimum Effect" and the "Amplitude-Descending Time Effect" are equally apparent in the ISN, MISN and GSN.

2. The use of MISN instead of $I S N$ slightly improves the correlation coefficient of the Waldmeier Effect and Amplitude-Period Effect. However, it slightly worsens the correlation coefficient of the Amplitude-Minimum Effect and Amplitude-Descending Time Effect.
3. If only $S C$ 10-22 are considered, then the correlation coefficients greatly worsen for all studied characteristics, except for the Amplitude-Descending Time Effect.

4. The Amplitude-Descending Time Effect must not be considered a tool for long-term solar activity prediction because the correlation between the amplitude of a solar cycle and the descending time three cycles earlier vanished when data of $S C$ 1-22 were used. Note that the exact relationship might be nonstationary. A similar conclusion may hold for Amplitude-Period Effect (see Table 4).

5. The use of MISN does not significantly improve the statistics of the characteristics of solar cycle.

Acknowledgements. Support from the Junta de Extremadura (Research Group Grant No. GR10131) and Ministerio de Economía y Competitividad of the Spanish Government (AYA2011-25945) is gratefully acknowledged.

\section{References}

Cameron, R., and M. Schüssler, Solar cycle prediction using precursors and flux transport models, ApJ, 659, 801-811, 2007.

Charbonneau, P., and M. Dikpati, Stochastic fluctuations in a Babcock-Leighton model of the solar cycle, Astrophys. J., 543, 1027-1043, 2000.

Chernosky, E.J., A relationship between the length and activity of sunspot cycles, Publ. Astron. Soc. Pac., 66 (392), 241-247, 1954.

Clette, F., D. Berghmans, P. Vanlommel, R.A.M. van der Linden, A. Koeckelenbergh, and L. Wauters, From the Wolf Number to the International Sunspot Index: 25 years of SIDC, Adv. Space Res., 40 (7), 919-928, 2007.

Dikpati, M., P.A. Gilman, and G. de Toma, The Waldmeier effect: An artifact of the definition of Wolf sunspot number? ApJ, 673, L99-L101, 2008.

$\mathrm{Du}, \mathrm{Z}$., and S. Du, The relationship between the amplitude and descending time of a solar activity cycle, Sol. Phys., 238 (2), 431437, 2006.

Du, Z.-L., H.-N. Wang, and X.-T. He, The relation between the amplitude and the period of solar cycles, Chin. J. Astron. Astrophys., 6, 489-494, 2006.

Faria, H.H., E. Echer, N.R. Rigozo, L.E.A. Vieira, D.J.R. Nordemann, and A. Prestes, A comparison of the spectral characteristics of the Wolf Sunspot Number $\left(R_{\mathrm{Z}}\right)$ and Group Sunspot Number $\left(R_{\mathrm{G}}\right)$, Sol. Phys., 223 (1-2), 305-318, 2004.

Getko, R., The intermediate-term quasi-cycles of Wolf Number and Group Sunspot Number fluctuations, Sol. Phys., 238 (1), 187206, 2006. 
Hathaway, D.H., R.M. Wilson, and E.J. Reichmann, Group Sunspot Numbers: Sunspot cycle characteristics, Sol. Phys., 211 (1), 357370, 2002.

Hoyt, D.V., and K.H. Schatten, Sunspot numbers: A new solar activity reconstruction, Sol. Phys., 179 (1), 189-219, 1998.

Karak, B.B., and A.R. Choudhuri, The Waldmeier effect and the flux transport solar dynamo, MNRAS, 410, 1503-1512, 2011.

Li, K.J., P.X. Gao, and T.W. Su, The Schwabe and Gleissberg Periods in the Wolf Sunspot Numbers and the Group Sunspot Numbers, Sol. Phys., 229 (1), 181-198, 2005.

Li, K.J., and H.F. Liang, Relationship between Group Sunspot Numbers and Wolf Sunspot Numbers, Astron. Nachr., 331 (7), 709-715, 2010.

Solanki, S.K., N.A. Krivova, M. Schüssler, and M. Fligge, Search for a relationship between solar cycle amplitude and length, Astron. Astrophys., 396, 1029-1035, 2002.
Svalgaard, L., Updating the historical sunspot record, in SOHO-23: Understanding a Peculiar Solar Minimum, eds. S.R., Cranmer, J.T. Hoeksema, and J.L. Kohl, ASP Conference Series, 428, 297306, 2010.

Vaquero, J.M., Historical sunspot observations: A review, $A d v$. Space Res., 40, 929-941, 2007.

Vaquero, J.M., and R.M. Trigo, Can the solar cycle amplitude be predicted using the preceding solar cycle length? Sol. Phys., 250 (1), 199-206, 2008.

Waldmeier, M., Die Zonenwanderung der Sonnenflecken, Astron. Mitt. Zürich, 14, 470-481, 1939.

Wilson, R.M., D.H. Hathaway, and E.J. Reichmann, An estimate for the size of cycle 23 based on near minimum conditions, J. Geophys. Res., 103 (A4), 6595-6603, 1998. 